\title{
DIFFUSION EQUATIONS FOR NONHOMOGENEOUS MEDIA. EXISTENCE OF SIMILARITY SOLUTIONS
}

\author{
F. ROMERO, J.L. ROMERO and J.F.R. ARCHILLA \\ Departamento de Fisica Térica, Facultad de Fisica, P.O. Box 1065. Seville. Spain
}

Kecerved 23 May 1985: accepted for publication 28 June 1985

We study the invariance of the diffusion equation $\delta P(x, t) / \delta t=(\delta / \delta x)[D(x) \delta P(x, t) / \delta x]$ under continuous groups of transformations. We show the conditions which $D(x)$ must satisfy for the existence of similarity solutions.

Recently considerable attention has been paid to the problem of diffusion in a medium whose diffusion coefficient varies in space. In one dimension $x$, it may be necessary to study the equation

$\frac{\partial P(x, t)}{\partial t}=\frac{\partial}{\partial x}\left(D(x) \frac{\partial P(x, t)}{\partial x}\right)$

where $P(x, t)$ represents the probability density for a diffusing particle to be at the point $x$ at time $t$, and $D(x)$ gives the value of the diffusion coefficient at each point $x$.

There are many situations in physics where this problem must be studied. As an example we shall mention the diffusion of hot electrons in velocity space $[1,2]$. There are no general solutions for this kind of problem, and special difficulties arise when contour behaviours must be taken into account.

In this paper we show that for a large class of diffusion coefficients, $D(x)$, it is possible to obtain exact solutions for eq. (1).

A possible way for obtaining exact solutions, called similarity solutions, for a given differential equation is by investigating its invariance under continuous groups of transformations $[3,4]$. Similarity solutions for eq. (1) could be obtained only for certain forms of $D(x)$.

We think that it is important to establish the conditions which $D(x)$ must satisfy in order to assure the existence of similarity solutions. Thus, if for a given problem one has a particular form of $D(x)$, it may be possible to check immediately if there exist similarity solutions. If that is the case, one may benefit from this solution method.

Eq. (1) enters in the context of a more general equation, usually called the Fokker-PlanckSmoluchowski equation (FPS):

$$
\begin{gathered}
\frac{\partial P(x, t)}{\partial t}=\frac{\partial^{2}}{\partial x^{2}}[a(x) P(x, t)] \\
-\frac{\partial}{\partial x}[b(x) P(x, t)] .
\end{gathered}
$$

This equation may be written in the form:

$H\left(x, P, P_{x}, P_{x x}, P_{t}\right) \equiv \alpha P_{x x}+\alpha P_{x}+\beta P-P_{t}=0$,

where

$\alpha=2 a^{\prime}-b, \quad \beta=a^{\prime \prime}-b^{\prime}$.

We introduce the group of transformations in the space $(x, t, P)$ given infinitesimally by

$x^{*}=x+\xi(x, t, P) \delta \epsilon$,

$t^{*}=t+\tau(x, t, P) \delta \epsilon$,

$P^{*}=P+\eta(x, t, P) \delta \epsilon$,

where $\epsilon$ is a continuous parameter and $\xi, \tau, \eta$ are called the infinitesimals of the group of transformations.

If $a(x)$ and $b(x)$ are such that it is possible to find the infinitesimals for eq. (3) to be invariant under the group of transformations, then there exist similarity 
solutions, which may be obtained by solving the characteristic equation

$\delta x / \xi(x, t . P)=\delta t / \tau(x, t, P)=\delta P / \eta(x, t, P)$.

It is not difficult to show that the infinitesimals which leave the FPS equation invariant have the following dependence:

$\xi=\xi(x, t), \quad \tau=\tau(t)$,

$\eta=f(x, t) P(x, t)+g(x, t)$,

where $\xi, \tau, f$ and $g$ are functions which must satisfy the following determining equations:

$a g_{x x}+\alpha g_{x}+\beta g-g_{t}=0$,

$a f_{x x}+\alpha f_{x}+\xi \beta^{\prime}+\beta \tau^{\prime}-f_{t}=0$,

$a\left(2 f_{x}-\xi_{x x}\right)+\alpha^{\prime} \xi+\alpha\left(\tau^{\prime}-\xi_{x}\right)+\xi_{t}=0$,

$a^{\prime} \xi+a\left(\tau^{\prime}-2 \xi_{x}\right)=0$.

Eq. (1) corresponds to eq. (2) for

$a=D(x), \quad \alpha=D^{\prime}(x), \quad \beta=0$.

With these identifications, eqs. (10)-(13) become:

$D^{\prime} g_{x}+D g_{x x}-g_{t}=0$,

$D f_{x x}+D^{\prime} f_{x}-f_{t}=0$.

$D\left(2 f_{x}-\xi_{x x}\right)+D^{\prime}\left(\tau^{\prime}-\xi_{x}\right)+\xi D^{\prime \prime}+\xi_{t}=0$,

$D\left(\tau^{\prime}-2 \xi_{x}\right)+\xi D^{\prime}=0$.

This system of equations is more easily treated if we introduce the variable:

$\bar{x}=\int_{x_{0}}^{x} \frac{1}{D(z)} \mathrm{d} z$.

In this variable, eq. (18) is a linear differential equation. Integrating it we obtain:

$\xi=\sqrt{D}\left(C+\frac{1}{2} \tau^{\prime} \bar{x}\right)$,

where $C$ is an arbitrary constant.

Here $C$ represents the invariance of translation in $\bar{x}$. We can take $C=0$ because any condition relative to the solutions in determined values of $\bar{x}$ (and then of $x$ ) avoids the invariance of translation.
If we substitute (20) into (17), and introducing the auxiliary function $H=\ln D$. we obtain after integrating:

$f(x, t)=-\frac{1}{8} \tau^{\prime \prime} \ddot{x}^{2}-\frac{1}{8} \tau^{\prime}\left(H_{\bar{x}} \bar{x}\right)+q(t)$,

where $q(t)$ is some function of $t$.

In the new coordinates $(\bar{x}, t)$ eq. (16) becomes

$f_{\bar{x} \bar{x}}+\frac{1}{2} H_{\bar{x}} f_{\bar{x}}-f_{t}=0$.

Subst ituting (21) into (22), we obtain

$\frac{1}{8} \tau^{\prime \prime \prime} \bar{x}^{2}+\left[-\frac{1}{4} \tau^{\prime \prime}-q^{\prime}(t)\right]-\frac{1}{8} \tau^{\prime} r(\ddot{x})=0$.

where

$r(\bar{x})=\left(H_{\bar{x}} \bar{x}\right)_{\bar{x} \bar{x}}+\frac{1}{2} H_{\bar{x}}\left(H_{\bar{x}} \bar{x}\right)_{\bar{x}}$.

The existence of similarity solutions requires that $D(x), \tau(t)$ and $q(t)$ have to be related in the way indicated by eq. (23).

We distinguish the following two cases in which that equation is satisfied:

Case I.

$H=\ln D$ arbitrary, $\tau^{\prime}=0$ and $q^{\prime}=0$.

The infinitesimals are

$\bar{\xi}=0$,

$\tau=\tau_{0}$,

$\eta=q_{0} P$.

This case yields a similarity solution of the form

$P(\bar{x}, t)=P(\bar{x}) \exp \left(q_{0} t / \tau_{0}\right)$,

where $P(\bar{x})$ satisfies the ordinary differential equation:

$P_{\bar{x} \bar{x}}+\frac{1}{2} H_{\bar{x}} P_{\bar{x}}-q_{0} / \tau_{0}=0$.

Of course it is well stablished that eq. (1) admits solutions in the form of separate variables. For this case our method does not give new results.

Case II.

$\tau^{\prime \prime \prime}=\mu \tau^{\prime}, \quad q^{\prime}=-\frac{1}{4} \tau^{\prime \prime}+\frac{1}{8} \kappa_{1} \tau^{\prime}$,

and

$G_{\bar{x} \bar{x}}+\frac{1}{2} \bar{x}^{-1} G_{\bar{x}} G-\mu \bar{x}^{2}+\kappa_{1}=0$,

where $\mu, \kappa_{1}$ are arbitrary constants and $G=\left(D_{\bar{x}} / D\right) \bar{x}$.

II.A. If $\mu=0$, the infinitesimals are (for the sub- 
group $g=0$ ):

$\xi=\left(\tau_{1} t+\tau_{2}\right) \bar{x}$,

$\tau=\tau_{1} t^{2}+2 \tau_{2} t+\tau_{0}$,

$\eta=P\left(-\frac{1}{4} \tau_{1} \bar{x}^{2}-\frac{1}{4}\left(\tau_{1} t+\tau_{2}\right) G+q\right)$,

with

$q=\frac{1}{8} \kappa_{1} \tau_{1} t^{2}+\frac{1}{4}\left(\kappa_{1} \tau_{2}-2 \tau_{1}\right) t+q_{0}$,

where $\tau_{1}, \tau_{2}, \tau_{0}$ and $q_{0}$ are arbitrary constants.

II. B. If $\mu \neq 0$, the infinitesimals are (for $g=0$ )

$\tau=\tau_{1} \exp (\sqrt{\mu} t)+\tau_{2} \exp (-\sqrt{\mu} t)+\tau_{3}$,

$\bar{\xi}=\frac{1}{2} \sqrt{\mu}\left[\tau_{1} \exp (\sqrt{\mu} t)-\tau_{2} \exp (-\sqrt{\mu} t)\right] \bar{x}$,

$\eta=P\left(-\frac{1}{8} \tau^{\prime \prime} \bar{x}^{2}-\frac{1}{8} \tau^{\prime} G+q(t)\right)$,

with

$q=q_{1} \exp (\sqrt{\mu} t)+q_{2} \exp (-\sqrt{\mu} t)+q_{3}$,

where $\tau_{1}, \tau_{2}, \tau_{3}$ and $q_{3}$ are arbitrary constants, and $q_{1}=\frac{1}{8} \tau_{1}\left(\kappa_{1}-2 \sqrt{\mu}\right), \quad q_{2}=\frac{1}{8} \tau_{2}\left(\kappa_{1}+2 \sqrt{\mu}\right)$.

Eq. (31) gives the condition that the diffusion coefficient $D(x)$ must satisfy so that eq. (1) admits similarity solutions. These could be obtained by solving the characteristic equation (8) with the infinitesimals associated. We are now working in order to find the solutions corresponding to some physical interesting cases which can not be resolved by other more elementary methods.

\section{References}

[1] P.I. Price, in: Fluctuation phenomena in solids, ed. R.E. Burgess (A cademic Press, New York, 1965).

[2] S.V. Gantsevich, V.L. Gurevich and R. Katilins, Riv. Nuovo Cimento 2 (1979) 1.

[3] L.V. Ovsjannikov, Gruppovye Svoystva Differentsialny Uravneni (Novosibirsk, 1962) [Group properties of differential equations, translated by G. Bluman (1967)] .

[4] G.W. Bluman, Construction of solutions to partial differential equations by the use of transformation groups, Ph.D. Thesis, California Institute of Technology (1967). 